\title{
Coordinated TEM and NanoSIMS of Presolar Graphites
}

T.K. Croat, F.J. Stadermann, and T.J. Bernatowicz*

* McDonnell Center for Space Sciences and Physics Department, Washington University, Saint Louis, MO 63130

Presolar graphites are especially interesting in that they encapsulate many internal grains during their growth, preserving these higher temperature phases that condense in the outflows of supernova (SN) and carbon stars. Coordinated laboratory microanalysis of these phase assemblages, using transmission electron microscopy and then secondary ion mass spectrometry with high spatial resolution (NanoSIMS), can reveal details about astrophysical processes that cannot be obtained through astronomical observations. Figure $1 \mathrm{a}$ and $1 \mathrm{~b}$ show a bright-field TEM and a NanoSIMS image of the same ultramicrotomed graphite slice, with the position of internal titanium carbides (TiCs) indicated with arrows. The internal TiCs are clearly visible in the NanoSIMS image due to the high ${ }^{16} \mathrm{O}$ signal from these regions. Not only was the ${ }^{16} \mathrm{O}$ signal higher from the TiCs, but the oxygen isotopic ratios from the TiCs were more anomalous than in the surrounding graphite [1]. This demonstrated ability to determine the isotopic composition of sub-micron phases along with the mineralogy yields detailed information about the formation history of presolar graphites.

Transmission electron microscopy of such ultramicrotomed graphite slices have revealed numerous internal grains, mainly refractory carbides (TiC) but also a number of rare phases such as kamacite, taenite, iron carbide $\left((\mathrm{Fe}, \mathrm{Cr})_{7} \mathrm{C}_{3}\right)$, metallic ruthenium-iron and metallic osmium. The loosely-structured turbostratic graphites are apparently quite effective at capturing and preserving condensates from the gas that formed at higher temperatures. The phase condensation sequence inferred from these phase assemblages can give insight into the condensation process of these presolar grains in the gaseous outflows from various types of stars. For example, figure 2 shows a titanium carbide with two attached kamacite grains which was then encapsulated within a graphite with the isotopic signatures of a supernova. The condensation sequence here is clearly $\mathrm{TiC} \rightarrow$ kamacite $\rightarrow$ graphite. When interpreted along with thermochemical models, this condensation sequence implies an iron abundance that is considerably above the solar ratios in the gas from which this graphite condensed [2].

The large number of internal carbides in some graphites (over 500 carbides within a single graphite Ke $3 \mathrm{e} 11$ ) enables an exploration of trends in grain size and composition as a function of radial distance from the graphite center (which is also a rough measure of the time at which grains accreted). Figure 3a shows a single slice of a SN graphite with many internal carbides, showing two regions with higher carbide density (central yellow region and exterior violet region) separated by a gap that contains few carbides (shaded blue). As shown in fig 3b., the two groups of carbides also have a clearly different chemical composition, with higher vanadium content in carbides towards the graphite interior. This graphite shows clear indications of changes in the gas composition during its growth. NanoSIMS investigations have not yet been done on these graphites, and these may reveal isotopic trends that mirror the chemical trends observed.

\section{References:}

[1] F.J. Stadermann et al., Geochim. Cosmochim. Acta 69 (2005) 177.

[2] T.K. Croat et al., Geochim. Cosmochim. Acta 67 (2003) 4705. 

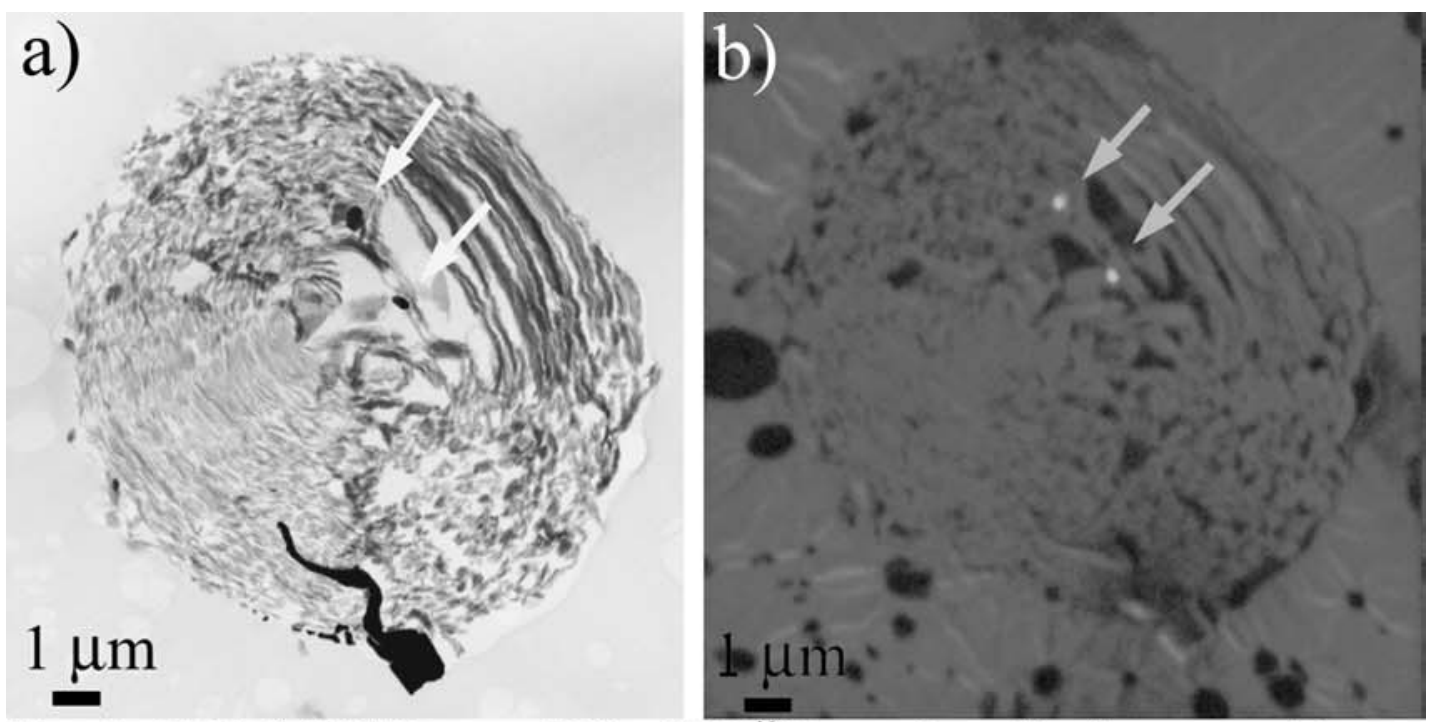

Figure 1. a) bright-field TEM image and b) NanoSIMS ${ }^{16} \mathrm{O}$ image of a graphite of supernova origin. Two internal TiCs are visible in both images (with positions indicated by arrows).

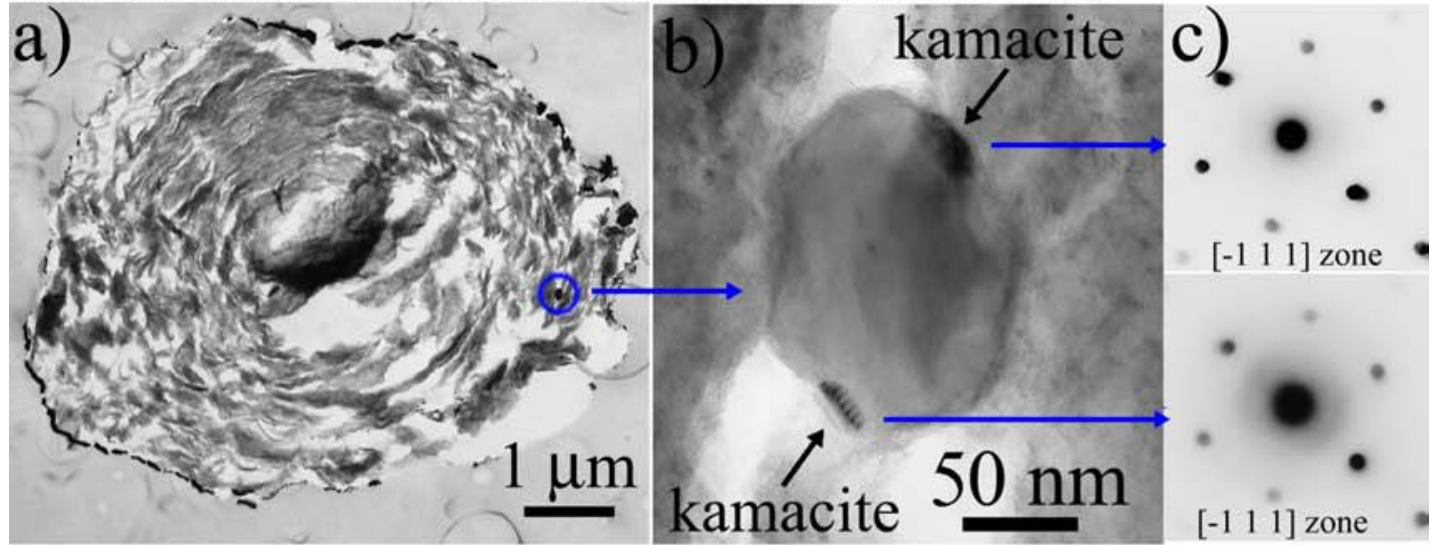

Figure 2. Bright-field TEM images of: a) sliced graphite and b) internal TiC with epitaxially-grown kamacites on opposite TiC crystal faces. Microdiffraction patterns (c) from the [ $\left.\begin{array}{lllll}-1 & 1 & 1\end{array}\right]$ zone of kamacite at the same relative orientation are also shown.
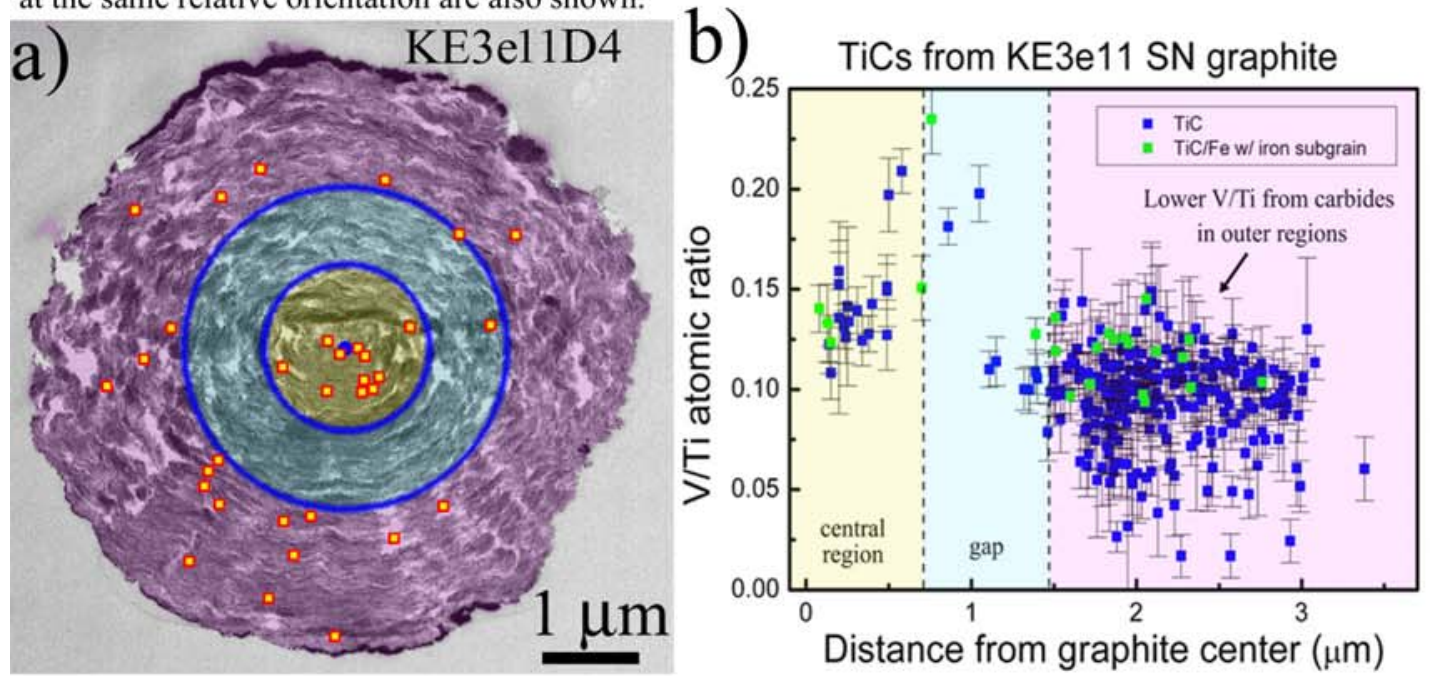

Figure 3. a) TEM image of sliced graphite with many internal TiCs and $\mathrm{TiC} / \mathrm{Fe}$ grains (at positions indicated by symbols). The image is divided into central and exterior zones with high carbide densities and an intervening gap of lower density. b) V/Ti atomic ratio from EDXS of all internal carbides as a function of distance from the center, showing different compositions between the two groups. 